\title{
Avec Yves Bonnefoy. De la poésie, par Jean-Paul Avice, Michael Edwards, Marc Grinberg, John Jackson, François Lallier, Patrick Née
}

\section{Mario Richter}

\section{(2) OpenEdition Journals \\ Edizione digitale \\ URL: http://journals.openedition.org/studifrancesi/35628 \\ DOI: 10.4000/studifrancesi.35628 \\ ISSN: 2421-5856 \\ Editore \\ Rosenberg \& Sellier}

\section{Edizione cartacea}

Data di pubblicazione: 1 juillet 2005

Paginazione: 123-124

ISSN: 0039-2944

\section{Notizia bibliografica digitale}

Mario Richter, «Avec Yves Bonnefoy. De la poésie, par Jean-Paul Avice, Michael Edwards, Marc Grinberg, John Jackson, François Lallier, Patrick Née», Studi Francesi [Online], 145 (XLIX | I) | 2005, online dal 01 novembre 2015, consultato il 18 avril 2021. URL: http://journals.openedition.org/studifrancesi/35628; DOI: https://doi.org/10.4000/studifrancesi.35628

Questo documento è stato generato automaticamente il 18 avril 2021.

\section{cc) (†)}

Studi Francesi è distribuita con Licenza Creative Commons Attribuzione - Non commerciale - Non opere derivate 4.0 Internazionale. 


\title{
Avec Yves Bonnefoy. De la poésie, par Jean-Paul Avice, Michael Edwards, Marc Grinberg, John Jackson, François Lallier, Patrick Née
}

\author{
Mario Richter
}

\section{NOTIZIA}

Avec Yves Bonnefoy. De la poésie, par Jean-Paul Avice, Michael Edwards, Marc Grinberg, John Jackson, François Lallier, Patrick Née, sous la direction de François Lallier, Presses Universitaires de Vincennes, 2001, pp. 130.

1 L'opera, organizzata da F. Lallier, è un omaggio offerto a Yves Bonnefoy da una pléiade di sette amici e estimatori (traduttori, poeti e critici, di ciascuno dei quali è fornita, in conclusione, una breve informazione bio - bibliografica).

2 La inaugura una pagina inedita, insieme alta e drammatica, dello stesso poeta (Aller encore), che si rivolge con trepidazione e affetto ai compagni di viaggio, da lui ritenuti alle prese con la sua medesima perigliosa e coraggiosa navigazione.

Il poeta inglese Michael Edwards (Les lieux de l'amitié) risponde all'amico col ricordo di un altro viaggio compiuto con lui per recarsi a visitare St Peter-on-the-Walls, nell'“Angleterre profonde", quasi immagine de L'Arrière-pays, viaggio conclusosi con una lettura di poesie dello stesso Bonnefoy, "lui qui est pour moi - scrive Edwards - la voix de la poésie française contemporaine et dans les poèmes de qui j'entends la voix de la poésie française elle-meme".

4 Jean-Paul Avice (La Poésie par c(l!ur), trae spunto da Terre seconde per introdurci in una articolata e ricca riflessione sul significato della poesia di Bonnefoy, che ha scelto la lettura selvaggia di adolescenti di borgata per giustificare che la terra comincia nelle parole una seconda esistenza, un'autentica 'incarnazione'. 
5 È bello e istruttivo seguire le considerazioni dedicate con sobrietà e intelligenza da Marc Grinberg, traduttore russo di Bonnefoy, allo stretto rapporto che lega l'“altérité" all'“amitié".

6 Seguono le interessanti e appassionate testimonianze-ricordi-riflessioni del curatore del libro, François Lallier (Etre présent à la présence), il cui rapporto con Bonnefoy dopo il 1968 trae senso dalla "présence" e dalla "vérité de parole". Non nella scrittura ma con la scrittura di Bonnefoy, afferma in conclusione l'autore, ha potuto egli ricevere delle enunciazioni di verità, dei "feux - precisa - qui sont évidence difficile mais joyeuse, gagnée sur le plan obscur, sur la volonté de mort, sur l'inconscient et sa cloture, et à la piace de l'Autre, qui est sans nom".

7 Nel suo breve e commosso intervento, John E. Jackson (Paroles de vie) confessa di dovere a Yves Bonnefoy, suo insostituibile amico, «quelques - unes des paroles les plus libératrices qu'on [lui] ait jamais dites».

8 L'ampio e articolato intervento di Patrick Née (Savoir lire, mais pour lever les yeux du livre) ci fa, in estrema sintesi, sapere che la parola di Bonnefoy non ci porta in alto o in basso per poi lasciarci soli di fronte al disgusto del reale, ma che essa agisce accompagnandoci «comme un viatique qui, à chaque degré dans la spirale, permet de montrer aussi bien que de descendre au long d'une initiation à double révolution du mystère d'être au monde, où l'on n'oublie jamais qu'on a pour tache d'essayer de mieux se comprendre pour communiquer à autrui, et transformer les limites qui nous sont imparties, de sexe, de nacissisme et finalement de mort inévitable - ou encore de structure psychique qui est le donné comme tel d'une éducation. et d'une histoire - en conditions d'appui, en forces d'appropriation mieux reconnues pour l'acceptation de soi, et par conséquent pour la reconnaissance du fait humain en général, jusqu'à ses plus fécondes, ou 'amoureuses', réalisations».

9 L'agile libro si conclude con un denso, straordinario intervento critico, ancora inedito, dello stesso Bonnefoy (Comment interpréter les peintures noires 1), intervento che prelude a un saggio che il poeta si propone di dedicare prossimamente a Goya. Si tratta di pagine sull'ultimo Goya, tratte da una conferenza pronunciata da Bonnefoy nel marzo $200 \mathrm{l}$ al Museo del Prado. Prendendo le mosse da un intenso e suggestivo esame dell'autoritratto ex-voto del pittore spagnolo intitolato Goya curato dal dottore Arrieta (del 1920), il poeta ci conduce - alla luce della "compassion" e del "dévouement désinteressé" - nei meandri segreti della psiche del pittore, autore, dopo la malattia, delle "peintures noires". Il poeta ci fa in tal modo conoscere la funzione liberatoria dello sfondo nei rapporti col primo piano. Nello sfondo si celerebbe la possibilità di accedere al puro pensiero di sé, a una sorta di vuoto della coscienza, essendo questa sottratta ai più diversi inganni dell'intelletto e delle illusorie sublimazioni. Questo sfondo finisce con l'imporsi drammaticamente, dice Bonnefoy, nella sua presenza assoluta, strenuamente realistica, con l'abissale quadro intitolato Un cane, | espressione piena dell'autentica compassione. 